\title{
Percepción del uso de TIC en las prácticas pedagógicas de los profesores de Educación Especial
}

\section{Perception on the Use of ICTs in the Teaching Practices of Special Education Teachers}

\author{
Raquel A. Aburto Godoy ${ }^{l}$ \\ Centro de Formación Continua \\ Universidad del Bio Bio, Sede Chillán \\ Chile \\ aburtogodoy@gmail.com
}

Recibido 08 de julio de 2011 • Aceptado 26 de agosto de 2011

\begin{abstract}
Resumen. El siguiente artículo tiene por finalidad exponer los resultados de la aplicación de un cuestionario sobre la percepción y uso de recursos tecnológicos dentro de las prácticas pedagógicas de los profesores de educación especial que se desempeñaban en grupos diferenciales de la comuna de Chillán, Chile, durante el año 2009. La aplicación de este cuestionario se hizo mediante la herramienta 2.0 Google Docs, en donde los profesores plasmaron sus respuestas, las que más tarde fueron tabuladas y analizadas mediante pruebas estadísticas con un diseño descriptivo. El cuestionario aplicado a los profesores de grupos diferenciales fue creado y validado para la presente investigación, lo que pone a disposición de la comunidad científica una herramienta precisa y de fácil aplicación.
\end{abstract}

Palabras claves. Educación especial, TIC, profesor, percepción, tecnología.

Abstract. This paper includes the results of a questionnaire on the perception and use of technological resources in the teaching practices developed by special education teachers who worked with differentiated groups in the community of Chillán, Chile, during 2009. This questionnaire was applied through the 2.0 Google Docs tool. The answers provided by the participant teachers were analyzed through statistical tests, with a descriptive design. The questionnaire applied was created and validated for this research; therefore, this research project provides an accurate, easy-to-apply tool for the scientific community.

Keywords. Special education, ICTs, teacher, perception, technology.

\footnotetext{
Doctora en Educación y Comunicación en Entornos Virtuales (c), Máster en Informática Educativa por la Universidad de la Frontera, Chile; Máster en Ciencias de la Educación especialidad en Tecnología Educativa de la Universidad Técnica de Lisboa, Portugal; Máster en Ciencias Humanas y Sociales mención Información y Comunicación de la Universidad de Poitiers, Francia. Ingeniera en Informática del Instituto Profesional Diego Portales, Chillán, Chile. Profesora de Educación Primaria con especialidad en Trastornos del Aprendizaje y Retardo Mental de la Universidad del Bio Bio, Chile. Trabaja actualmente como Asesora Pedagógica en el Centro de Formación Continua de la Universidad del Bio Bio, Sede Chillán, Chile. Ha desempeñado docencia en áreas de educación especial, informática y básica general.
} 


\section{Introducción}

A raíz de la tremenda rapidez con que las TIC evolucionaron, la educación está viviendo un desajuste entre lo "que es" y lo "que debería ser", puesto que no se han implementado prácticas pedagógicas capaces de tratar a los estudiantes de la "Generación Net" (Tapscott, 1999 citado por Ministerio de Educación de Chile [MINEDUC], 2007) o los "Nativos Digitales" llamados así por Prensky (2001, p. 1), quienes:

Antes de entrar a la universidad poseen 10 mil horas de videojuego, 200 mil mails mandados y recibidos, 10 mil horas de uso de celular, 20 mil horas de TV y finalmente, 5 mil horas de lectura. Es decir, una generación capaz de absorber información y tomar decisiones muy rápido, con procesamiento paralelo, que lee formato hipertexto ${ }^{2}$.

Es importante detenerse a pensar lo que significa para esta generación estar sentados, frente a una clase expositiva, donde el rol protagónico corresponde al profesor y cuya única tarea para ellos es mantener la atención y escuchar. A este entorno debemos agregarle las diferencias en los estilos de aprendizaje, las experiencias personales y las necesidades educativas especiales (NEE) (Vázquez y Fernández, 2003). Estos autores también sostienen que es aquí donde nace la importancia de incorporar nuevos elementos tecnológicos al proceso educativo, los que permitan atender los múltiples requerimientos tanto de los estudiantes como de los contenidos y, en general, de la era actual, pero no solo para conseguir objetivos pedagógicos, sino para ser utilizados en todo el proceso.

Mc Clintock (1993) vislumbra las ventajas de la utilización del ordenador como sistema, no solo dentro del mundo educativo sino en todo los ámbitos sociales, valora la introducción de una nueva forma de representación de la información, una nueva manera de codificar las cosas: la digitalización de la información.

Esto plantea un desafío para los docentes, quienes deberán replantearse los roles en los que generalmente ha venido reposando la práctica docente, instándoles a apoyarse en otros recursos para la detección y análisis de los niveles de aprendizaje de los estudiantes.

Está claro que los lineamientos a seguir para la formación de los docentes deben cambiar al ritmo de los necesidades actuales. Por una parte, se debe habilitar a todos los docentes en ejercicio para manejar herramientas tecnologías de información y comunicación (TIC) que les permitan optimizar los tiempos en la gestión docente, se valoran, así, las aportaciones de estas, para luego incluirlas dentro de su función pedagógica (Vázquez y Fernández, 2003).

Por otro lado, se deben reestructurar las mallas curriculares de las instituciones formadoras de docentes para incluir dentro de estas aspectos del conocimiento de TIC relacionados con su utilidad en los procesos de aprendizaje y manejo de la información. En ambos casos, el fin último es que los docentes, de todos los niveles y especialidades, se impregnen con la tecnología; que les resulte fácil y natural incorporarla a su trabajo docente, desarrollando lo que se ha llamado una "visión técnico-pedagógica", es decir, buscar el sustento pedagógico y las habilidades que ciertos elementos TIC pueden desarrollar en los alumnos (MINEDUC, 2007).

\footnotetext{
Traducción de la autora.
} 
Esta idea se refuerza con el pensamiento de McClintock (1993, p. 12) quien plantea que "los educadores no se pueden resistir a las nuevas tecnologías siempre que estas tengan características adecuadas a los propósitos de la educación, especialmente los que se refieren a permanencia en el tiempo (...)".

Por su parte, Semenov (2006, p. 17) concibe que las TIC puedan ayudar a los educadores a construir un tipo de sociedad multicéntrica y multicultural, ya que permiten:

- Fomentar el éxito personal sin ensanchar la brecha entre los más pobres y más ricos.

- Apoyar modelos de desarrollo sostenible.

- Ayudar a que una cantidad mayor de países construyan y utilicen un espacio de información, y no que unos pocos países y monopolios de medios de comunicación masiva dominen la transición de información y la difusión de patrones culturales.

"Estamos seguros de que las TIC serán una herramienta fundamental para favorecer un cambio positivo, siempre y cuando se utilicen de forma creativa y con vistas al bien común" (Semenov, 2006 p. 16). Al respecto, no se debe perder el norte de reconocerlas como recursos pedagógicos, sin olvidar que la genialidad de estas se encuentra en la creatividad del docente.

Para la Educación Especial, los recursos tecnológicos proporcionan un sinfín de funcionalidades que van desde instrumentos de ayuda técnica, a instrumentos de diagnóstico y re-educación. En esta idea, Soto (s. f.) sostiene que cada vez son más las personas que encuentran en las tecnologías un punto de apoyo para su desarrollo: los alumnos para compensar discapacidades, y los profesores, para alcanzar su máximo desarrollo profesional y potenciar el desarrollo cognitivo de los alumnos, al mejorar los procesos de adquisición de los objetivos de las distintas disciplinas del currículo escolar.

\section{Evolución histórica de la Educación Especial}

La evolución de la Educación Diferencial mundial ha estado impulsada por grandes esfuerzos para abrir espacios a las personas con NEE (necesidades educativas especiales), por tanto, revisaremos dos de los hechos más relevantes en esta área, a nivel internacional.

Con el informe The Warnock Report (1978), se inicia una nueva forma de entender la Educación Especial. Este informe, desarrollado en Inglaterra, afirma que los fines de la educación son los mismos para todos los niños y niñas, cualesquiera sean los problemas con que se encuentren en sus procesos de desarrollo. En consecuencia, la educación es un continuo de esfuerzos para dar respuesta a las diversas N.E.E de los alumnos, en el propósito de alcanzar los fines propuestos. Dieciséis años más tarde, en 1994, representantes de 92 gobiernos y 25 organizaciones internacionales se reunieron en Salamanca, España, del 7 al 10 de junio, con el fin de promover el objetivo de la Educación para Todos, aprobando la Declaración de Salamanca. Este documento está inspirado por la integración y el reconocimiento de la necesidad de actuar con miras a conseguir "escuelas para todos", esto es, instituciones que incluyan a todo el mundo, celebren las diferencias, respalden el aprendizaje y respondan a las necesidades de cada cual. 
En conjunto con estos esfuerzos, la evolución histórica de la Educación Especial ha pasado por varias fases (Gento, 2003): eliminación, beneficencia, educación y tratamiento pedagógico. A continuación una síntesis de cada una de ellas:

\subsection{Fase de eliminación}

Esta fase está dada principalmente por la indiferencia y rechazo hacia los niños que presentaban algún tipo de deficiencia. Por ejemplo, en el pueblo espartano, la Ley de Licurgo establecía que los más ancianos debían revisar a todos los recién nacidos para determinar si tenían algún tipo de debilidad, de ser así, eran lanzados por el monte Taijeto. Igual situación ocurría con los griegos y romanos, aunque estos, en algunos casos, dejaban vivir a estos sujetos para utilizarlos dentro de sus espectáculos. Con la llegada del Cristianismo esta situación se tornó más cruda, ya que se consideraban sus taras como un castigo divino o demonios interiores, en el caso de los epilépticos.

\subsection{Fase de beneficencia}

Fueron las órdenes religiosas las primeras en presentar algún interés en este tipo de sujetos mitad endemoniados, mitad delincuentes. Es así que por el siglo XV, Fray Gilabert Jofre fundó, alrededor de 1409, el "Hospitals dels Ignoscents", considerado como el hospital psiquiátrico más antiguo del mundo por los historiadores de la medicina. Más tarde, en Valencia (España), surge la llamada "Casa de los Oficios" en la cual trabajaban enfermos mentales. Es la primera terapia ocupacional que se conoce en la historia.

\subsection{Fase de educación}

En el siglo XVI aparecen las primeras experiencias pedagógicas, como es el caso de Vicente de Paúl, quien fundó diversos centros en los que se trataban a personas deficientes (retrasados, ciegos, lisiados, entre otros). Fray Pedro Ponce de León trabajó en España con deficientes auditivos creando el Método oral. Juan Pablo Bonet, en 1620, fue el autor del primer libro de especialidad titulado Reducción de las letras y el arte de enseñar a hablar a los mudos. En 1755 el abate francés L'Epée inventa un lenguaje de gestos y, con ellos, un alfabeto de signos para enseñar a hablar a los sordos, con una metodología que más tarde fue utilizada por Hauey y Braille para enseñar el alfabeto táctil a los ciegos. Es a finales de este siglo cuando aparece un importante cambio de actitud hacia los sujetos que presentan deficiencias, se pasa de tenerlos recluidos a prestarles atención médica. En general, se admite que las investigaciones en el campo de la educación de los deficientes se inician con la obra del francés Jean Itard (1774-1836) sobre Víctor, el niño salvaje de Aveyron.

Itard, Seguin, Esquirol, Bourneville y Samuel Howe, médicos y pedagogos, están considerados como los padres de la Educación Especial. A partir de sus trabajos, la Educación Especial surge como campo profesional, con pioneros tanto europeos como americanos (Gútiez, 2003). 


\subsection{Fase de tratamiento pedagógico}

En el siglo XX Montesano y Montessori fueron los pioneros en la asistencia médico-pedagógica a los deficientes mentales en Italia. En Suiza, Hanselman y Decroly constituyen los grandes modelos de la pedagogía terapéutica. Este último realizó una clasificación de niños retrasados ${ }^{3}$ e inventó juegos para estos, los cuales utilizó después con niños normales. En 1905, en Francia, Binet y Simon publican la primera escala del desarrollo intelectual al igual que una escala de pruebas que explicaban las variables de inteligencia existente entre los adultos retrasados, lo que permitió definir la idiotez, imbecilidad profunda y ligera. En esta época, aparece un gran interés por los trastornos del aprendizaje y se hace un gran énfasis en los problemas del lenguaje, al igual que se realizan intentos para definir la afasia. En 1922 se celebra el primer Congreso de Pedagogía Terapéutica. En 1937 se crea la Sociedad Internacional de la Infancia Deficiente y se inicia propiamente la Educación Especial en España y en el resto de Iberoamérica.

En 1963 se utiliza, por primera vez, el término Learning disabilities (traducido al español como "dificultades de aprendizaje") por Samuel Kirk durante la conferencia en Chicago, organizada por la Fund for Perceptually Handicapped Children, . Ese mismo día se formó la Association Children and Adults with Learning Disabilities (ACLD), asociación que ha tenido una gran influencia en el desarrollo de este campo, al exigir una regulación legal, recursos y servicios públicos que atendieran las demandas educativas de los alumnos con dificultades de aprendizaje.

\section{Una mirada a la Educación Especial en distintos países}

\subsection{La situación de la Educación Especial en África y Asia}

Según datos de Naciones Unidas, en el mundo hay más de 600 millones de personas con discapacidad (el 10\% de la población mundial), de las que 80 millones viven en África, donde tener un hijo con discapacidad es considerado una carga y un castigo. Sin embargo, es difícil obtener datos que puedan ilustrar la magnitud del problema, por la falta de recursos en el sector sanitario de este país. Además, la naturaleza de la pérdida de audición suele verse como una discapacidad oculta.

Los esfuerzos que se han desarrollado por apoyar la educación y capacitación de personas con déficit visual comenzó alrededor de 1963, en Senegal, donde se aprobó una resolución sobre la posibilidad de conceder becas a personas ciegas que fueran capaces de aprender Braille, la concesión de los equipos necesarios para la enseñanza a ciegos, el suministro de equipo necesario para uno o más talleres y la posibilidad de enviar maestros para ciegos a Senegal en virtud de la asistencia técnica.

En mayo de 1966, Senegal fue el destinatario de una nota del Secretario General de la Organización de la Unidad Africana sobre la creación de un Comité de Acción para los Ciegos en el África occidental. Más tarde en 1974, el Estado de Senegal firmó un acuerdo con la Fundación Estadounidense para los Ciegos de Ultramar, con lo cual obtuvo el envío de un experto maestro en la formación de treinta profesores durante dos años especializándose en la enseñanza de niños ciegos, así como la provisión de materiales didácticos.

Término que se empleaba en esta época para referirse a las personas que presentaban una discapacidad intelectual. 
Esta capacitación se llevó a cabo entre 1974 y 1976. Así empezó una gran empresa que ha permitido al sistema educativo ser más democrático y ha ofrecido a cientos de niños ciegos las oportunidades de vencer la oscuridad de la ignorancia, limitante de su discapacidad .

Las distintas clasificaciones sobre la condición de discapacidad que actualmente se están considerando en las políticas de gobiernos son: déficit visual, discapacidades físicas, deficiencias mentales, trastornos del lenguaje y dificultades de aprendizaje.

Por su parte en Asia, en 1995, según la Organización Mundial de la Salud (OMS), existían más de 25 millones de personas en el sudeste asiático que sufrían una pérdida de audición importante, datos corroborados por la Federación de Personas Discapacitadas de China (CDPF). Exactamente 20 millones es la cifra de los casos registrados por la CDPF. La cifra real de discapacitados auditivos en China puede ser mucho mayor. Según la CDPF, el $43 \%$ de los discapacitados auditivos chinos no pueden permitirse comprar audífonos por motivos económicos. En Tailandia, los datos indican que aproximadamente el 13,5 por ciento de la población padece pérdida auditiva. En Pakistán rural uno de cada 12 niños sufre pérdida de audición

\subsection{La situación europea en el área de la Educación Especial}

Dentro de la situación en la educación diferencial en Europa se van a destacar los programas Comenius y Jean Monnet.

En la Comisión Europea dentro del Programa Comenius se encuentra la acción dedicada a facilitar la implementación de actividades específicas para promover la integración de personas que presentan NEE, así como la promoción de la educación intercultural para luchar contra el racismo en los niveles de educación básica y media (primaria y secundaria).

En la educación universitaria, la situación europea en Educación Especial, actualmente, está estructurada por el Programa Jean Monnet, creado en 1990, el cual tiene por finalidad estimular la excelencia en la enseñanza, investigación y la reflexión sobre la integración europea en las instituciones de educación superior.

Este programa tiene tres actividades claves:

i. Apoyo a la Universidad en proyectos sobre la integración europea: Lo que incluye cátedras universitarias, centros de excelencia, módulos de cursos, información o actividades de investigación, así como el apoyo a asociaciones académicas de profesores e investigadores en la integración europea.

ii. Apoyo a seis instituciones académicas: Como el Colegio de Europa, el Instituto Universitario Europeo y la Agencia Europea para el Desarrollo de las NEE, ámbito de interés para esta investigación.

iii. Apoyo a nivel europeo a las asociaciones que trabajan en el ámbito de la educación y formación.

La Agencia Europea para el Desarrollo de las NEE (European Agency for Development in Special Needs Education) es una de las seis instituciones amparadas por el Programa de Acción de la Unidad Europea, fue creada en 1996 y se mantiene por los Ministerios de Educación de los países 
participantes (Estados miembros de la Unión Europea, así como Islandia, Noruega y Suecia) y está respaldada por la Comisión y el Parlamento Europeo. En su trabajo tiene en cuenta las declaraciones internacionales fundamentales sobre la Educación Especial, las Reglas Estándar de las Naciones Unidas, la Declaración de Salamanca, la Carta de Luxemburgo, las Resoluciones del Consejo de la UE concernientes a la educación inclusiva y la Declaración de Madrid.

Los objetivos fundamentales de la Agencia son:

a. Fomentar la calidad en el campo de las NEE, mediante el mantenimiento de un marco a largo plazo para extender la colaboración europea.

b. Ofrecer una reflexión responsable de las realidades de las NEE en Europa, con información actualizada que pueda relacionarse con los contextos nacionales de los países.

c. Identificar los factores claves que dificultan o apoyan las experiencias positivas.

d. Facilitar el intercambio efectivo de información y experiencia entre y dentro de los países miembros.

e. Facilitar a los responsables políticos y los profesionales el acceso a la información relevante, ofreciendo mecanismos y servicios que posibiliten compartir información y mejoren el contacto entre los distintos usuarios.

En el estudio La Educación Integradora en Europa, desarrollado por la Agencia para el desarrollo de las NEE en el año 2000, se analiza la tendencia de la situación en esta materia de 17 países europeos. Se recopilan los siguientes elementos: apoyo al profesorado y formación del profesorado.

a. El apoyo al profesorado: Los profesores de aula asumen la responsabilidad de todos los alumnos: Los profesores de aula se mencionan en todos los países como los profesionales responsables de todos los alumnos, incluyendo aquellos con necesidades educativas (Soriano, 2000). Esto hace que sea muy importante que los profesores de aula común reciban información y orientaciones necesarias sobre la diversidad de sus estudiantes.

b. Formación del profesorado: La Formación del profesorado común que trabaja con alumnos de NEE es un factor clave. La formación del profesorado necesita considerar que el profesor de aula es el profesional responsable de la educación de todo el alumnado. Necesitan recibir -durante su periodo inicial de formación- los conocimientos y las destrezas adecuadas para conocer y responder a la diversidad de las necesidades de los alumnos.

Todos los profesores reciben algún tipo de formación sobre educación especial durante su formación inicial: Todos los profesores, y en todos los países, reciben algún tipo de capacitación relacionada con la educación especial. El principal objetivo parece ser proporcionar conocimientos básicos para que el tutor pueda trabajar con todos los alumnos. Posteriormente se proponen diversas posibilidades de formación para ampliar o profundizar sus conocimientos y destrezas en áreas más específicas (Soriano, 2000). 
La formación complementaria es, por lo general, optativa y forma parte de la formación permanente: esta situación refuerza la idea de que los profesores deberían tener las destrezas necesarias al acabar su formación inicial.

Los cursos de formación permanente son optativos en todos los países: Se ofrecen bastantes posibilidades a los profesores dentro del marco de los recursos de formación. Puede incluir sesiones organizadas por centros de formación, universidades, autoridades educativas o colegios.

Según el estudio ECHP [Panel de Familias de la Comunidad Europea] solo el 9\% de las personas severamente discapacitadas y el $14 \%$ de aquellas con cierto grado de incapacitación llegan a la educación universitaria, en comparación con el 18\% de las personas sin discapacidades (Pérez, 2003, pp. 29-30).

De acuerdo con el Panel de Familias de la Comunidad Europea, una persona en edad activa (16-64 años) tiene una probabilidad del 66\% de encontrar un trabajo o desarrollar un negocio. Para una persona con una discapacidad leve la probabilidad se convierte en el $47 \%$ y para una persona discapacitada severa la probabilidad se reduce al $25 \%$.

El nivel de empleo de mujeres con discapacidad es considerablemente más bajo. Entre las mujeres con una discapacidad grave, solo el $25 \%$ está trabajando, lo cual muestra un porcentaje muy elevado de inactividad. Igualmente, solo el $44 \%$ de mujeres con una discapacidad moderada está trabajando.

Las personas con discapacidad son especialmente vulnerables a la exclusión social:

- Los ingresos medios por familia son sustancialmente menores en una familia con una persona con discapacidad.

- Existe una clara correlación entre la gravedad de la discapacidad y el grado de pobreza y exclusión.

- Las mujeres con discapacidad son víctimas frecuentes de la pobreza y la exclusión social.

- Las personas que viven en instituciones no padecen una pobreza financiera grave aunque experimentan una extrema exclusión social.

\subsection{La situación de algunos países de Iberoamérica en Educación Especial}

Según Díaz, Rojas y Vasco (2000), la situación iberoamericana en cuanto al proceso de configuración de la educación especial estuvo marcado por una primera etapa en la que se realizaron intentos por organizar instituciones especializadas a finales del siglo XIX hasta finales de la década de los 40, cuando se crean las primeras escuelas dedicadas especialmente a personas con discapacidad visual, con predominio de un servicio de tipo médico-asistencialista.

En este primer periodo se hace necesario comentar el caso de Colombia, donde se crean impuestos destinados a apoyar las instituciones de educación especial y, además, se asignan estímulos a los docentes que trabajan en esta área.

Otro caso importante de señalar es el de Paraguay, en este país en 1939 comienzan los programas de educación especial, sin embargo, a partir de 1954 es cuando, con la creación de 
escuelas diferenciales a cargo del Ministerio de Educación y Culto, realmente se inicia la educación diferencial sistematizada (Díaz, et al. 2000).

En 1910 se ubican los orígenes de la educación especial en Uruguay, se crea un establecimiento para sordomudos dependiente del Consejo de Educación Primaria y Normal, que luego se transformaría en la Escuela para discapacitados auditivos. Es hasta 1927 cuando comienza la atención para discapacitados intelectuales.

Bolivia, por su parte, ofrece ya algunos referentes en el año 1927, cuando inicia la enseñanza de la lecto-escritura a un joven ciego utilizando el sistema Braille. La consolidación de la Educación Especial en este país no es hasta la década de los 60, cuando se crea e integra una clase de esta materia en las escuelas normalistas del país.

Una segunda etapa, que comprende las décadas de los 50 y los 60, es considerada en muchos países como el auge de la educación especial, ya que aparecen las primeras instituciones para la atención de personas con discapacidad.

En este periodo, es importante señalar el caso de Costa Rica que comenzó con esfuerzos en centros especializados en los cuales se acogía a los alumnos en régimen de segregación. Sin embargo, en 1973 se iniciaron las denominadas actualmente "aulas integradas" en las escuelas del sistema regular $\mathrm{y}$, en los últimos quince años, se ha promovido la integración de los alumnos que presentan necesidades educativas generadas por una condición de discapacidad a la educación regular.

En el caso de Cuba, desde 1959 comenzó la atención para niños y jóvenes con deficiencias físicas y mentales. En los primeros años se crearon más de 50 escuelas y se establecieron capacitaciones para preparar y mejorar al personal docente.

También en Argentina por una fuerte influencia de las idea de O. Decroly y M. Montessori, donde se organizó la Educación Especial como un régimen especial del sistema educativo y se establecieron los mecanismos necesarios para atender las necesidades que no pueden ser satisfechas por la estructura básica del sistema regular.

\subsection{Educación Especial en Chile}

En el 2005, el Ministerio de Educación de Chile realiza un exhaustivo análisis de la evolución de la Educación Especial. A continuación un análisis de sus hitos más importantes.

La Educación Especial en Chile tiene sus orígenes hacia mediados de 1800, en el Gobierno del Presidente Bulnes. En 1852 se crea la primera escuela para sordos con el apoyo de Montt y Lastarria y dos años más tarde se funda la Escuela de Sordas, a cargo de Rosario Vargas; no obstante, estas escuelas tuvieron que cerrar siete años más tarde por déficit fiscal. Luego, con la llegada a Chile de dos profesores extranjeros, se crean dos escuelas: una para ciegos y otra para sordos con la supervisión de Adrian Farías.

Durante el gobierno del Presidente Federico Santa María, en 1883, se destinan fondos del gobierno para abrir nuevas escuelas, proporcionar libros y dar la posibilidad de que profesores se fueran a estudiar en el extranjero. En 1885 se abre, en Santiago, la escuela para enseñanza de sordomudos, guiada por las hermanas del buen pastor. 
Ya en 1900 se crea la escuela para sordos y un curso para no videntes y, tres años más tarde, se inaugura la escuela experimental para personas con déficit intelectual.

Se crea en el Ministerio de Educación la Jefatura de Educación Especial.

Otro hecho de envergadura en este periodo fue la creación de centros y microcentros de diagnóstico (hoy equipos multiprofesionales), junto con los grupos diferenciales. Otro importante aporte fue la creación de pos-títulos dirigidos a maestros normalistas para formarlos como profesores especialistas. Es así como en 1964 la Universidad de Chile crea el Centro de Formación de Especialistas en Deficiencia Mental y, más tarde, en 1966 y 1970, se abre el pos-título en audición y lenguaje, y trastornos de la visión, respectivamente.

Desde 1974 en adelante, diversas instituciones privadas crearon escuelas especiales, incorporando a la educación a más personas con discapacidad.

Durante los años 80, desde esta nueva perspectiva, se elaboran planes y programas de estudio para las distintas discapacidades (enfoque de habilitación o rehabilitación), programas vigentes hasta la fecha. En los 90, se inicia en Chile el proceso de reforma a la educación con el fin de lograr una mejora en calidad y equidad, se busca modernizar el sistema y garantizar respuestas educativas de calidad para todos y cada uno de los niños, niñas, jóvenes y adultos.

Entre 1992 y 1997, el Ministerio de Educación implementó la línea del Programa MECE/ BÁSICA, dirigido a profesores de escuelas especiales y de grupos diferenciales que permitió, luego de muchos años, que los profesores y especialistas se perfeccionaran en nuevos enfoques para abordar la atención de las NEE.

La cobertura a alumnos que presentan NEE, actualmente, se despliega de la siguiente manera:

1. Grupos diferenciales: Destinado para personas con necesidades educativas especiales, pero no derivadas de una discapacidad. El apoyo se realiza dentro de la misma unidad educativa a la que asiste el alumno, puede ser en una sala diseñada para tales efectos (sala de recursos) o dentro de la sala común con todos los otros estudiantes. El profesor de grupo diferencial debe proporciona estrategias pedagógicas diversas, las cuales deben servir como guía para el profesor de aula, en donde está el alumno que asiste a grupo diferencial. La cantidad de estudiantes atendidos mediante esta modalidad no debe exceder a 5 por curso.

2. Escuelas especiales: Ofrecen servicios educativos a aquellos niños con discapacidad intelectual, auditiva, visual, motora y graves alteraciones para comunicarse oralmente. Esta actúa en forma paralela con la escuela normal. Posee diferentes niveles y por lo general termina en una capacitación laboral.

3. Aulas y escuelas hospitalarias: Este beneficio lo obtienen aquellos niños de educación regular o especial que, por alguna razón de enfermedad, se encuentran hospitalizados ya sea por un tratamiento médico a alguna enfermedad crónica o tratamiento ambulatorio.

4. Proyecto de integración escolar: Un proyecto de integración escolar es una estrategia o medio de que dispone el sistema para incorporar un niño, niña o joven con discapacidad al sistema educativo regular. En el año 2006, la matrícula en programas de integración escolar ascendía a 48.948, en 2007 la cifra alcanza los 55.090; en el 2008, 64.361, en 2009, 72.032 y en 2010, la matrícula de integración escolar alcanzó 75.683 (MINEDUC, 2005). 


\section{Formación de profesores de Educación Especial}

Los estudiantes que asisten a los establecimientos educativos son cada vez más diversos, cada uno con experiencias variadas y con demandas diferentes al entorno escolar. En este sentido, no se pueden ignorar las diferentes competencias que al profesor actual se le exigen; los cambios tecnológicos y sociales requieren unos profesionales activos, con una actitud abierta a la formación permanente y a la innovación (De Martín, 2005, citado por Gallego y Rodríguez, 2007).

La Unesco (2011), en este sentido identifica cuatro valores, como base de las competencias esenciales que todos los docentes de educación especial deben poseer. Estos son:

- Valorar la diversidad del alumnado: Las diferencias de los alumnos son consideradas como un recurso y un activo de la educación.

- Apoyo a todos los alumnos: Los profesores deben tener altas expectativas de logros para todos sus alumnos.

- Trabajo en equipo: La colaboración y el trabajo en equipo son esenciales para todos los enfoques.

- Continuo en el desarrollo profesional personal: La enseñanza es una actividad de aprendizaje y los profesores deben aceptar la responsabilidad de su propio aprendizaje permanente.

Pavone (2009) plantea que el proceso de formación deberá estar caracterizado por un conjunto de diferentes posibilidades, como la formación cultural, la programación y metodología-didáctica, la observación de las clases que acogen a estudiantes con deficiencias. Es decir, se trata de apostar e invertir en un maestro de reflexión y no tan técnico, puesto que debe centrar su reflexión en los problemas reales y gente real que encontrará en su vida diaria (Sanches, 1995). La integración de estas diversas dimensiones no puede ser realizada a través de la simple integración de dominios o de diferentes contenidos de programas de estudio. La integración puede ser realizada favorizando un proceso de elaboración basado en la dialéctica y la confrontación de metodologías. En otros palabras, no es un programa académico, sino un proyecto de formación (Pavone, 2009).

\subsection{La importancia de la formación de los profesores en TIC}

Como fácilmente se acepta, una de las cuestiones centrales de la problemática de la integración de las TIC en el contexto educativo tiene que ver directamente con lo que los profesores sean capaces de hacer en el aula con sus alumnos. Esto estará supeditado no solo a factores de orden personal -como la motivación y la actitud-, sino -lo más importante- en la preparación de los profesores, esto es, en los conocimientos y competencias que, efectivamente, tienen sobre los modos de integración y exploración de las TIC en contextos específicos de enseñanza y aprendizaje en el que intervienen (Costa y Viseu, 2008). Está claro que la situación dentro de las escuelas y, específicamente dentro de las aulas, está lejos de ser considerada satisfactoria, ya que aún las universidades como entidades formadoras no tienen claro el tipo de formación que los profesores requieren para enfrentar con éxito las demandas, no solo de los estudiantes de la clase, sino de toda una sociedad. Es así que un profesor recién egresado ya se encuentra desfasado entre a la preparación recibida y las necesidades del entorno. Por lo tanto, urge incorporar en la 
formación docente inicial una seria de elementos relacionados a la inserción de las TIC en los procesos de enseñanza y aprendizaje que preparen a los docentes para los escenarios actuales. Uno de los referentes a seguir para esta incorporación de las TIC a la formación inicial de los docentes corresponde a un informe realizado por la UNESCO (2002), el cual plantea 4 grupos en los cuales se organizan las competencias a desarrollar en los profesores. A continuación un análisis de estas:

- Pedagogía: Es el aspecto más importante de la incorporación de la tecnología en el currículo. La pedagogía se centra tanto en la prácticas pedagógicas de los maestros, como en los conocimiento de los planes de estudio. Requiere, además, que en el desarrollo de aplicaciones dentro de sus disciplinas hagan uso efectivo de las TIC, para apoyar y extender la enseñanza y el aprendizaje. Por tanto, es fundamental, asociados con el objeto de su disciplina, el contexto local y el enfoque individual del docente.

- Colaboración y trabajo en red: El desarrollo de competencias docentes en redes y de colaboración son esenciales para las TIC en la educación. Mediante la colaboración y trabajo en red, los docentes promueven el aprendizaje democrático en el aula y se basan en los conocimientos locales y mundiales. El papel del profesor se expande al de un facilitador de la colaboración y la creación de redes con las comunidades locales y globales. La expansión de la comunidad de aprendizaje fuera del aula también exige el respeto a la diversidad, incluida la educación intercultural y equitativa y el acceso a los recursos electrónicos de aprendizaje.

- Asuntos sociales y sanitarios: La tecnología trae consigo nuevos derechos y responsabilidades, por lo que debe tener en cuenta el acceso equitativo a los recursos tecnológicos, el cuidado de la salud individual y el respeto de la propiedad intelectual, incluidos en el aspecto de competencia de asuntos sociales de las TIC. En concreto, es necesario que los docentes comprendan y apliquen los códigos legales y morales de la práctica, incluyendo los referidos a derecho de autor y el respeto de la propiedad intelectual; de igual manera, se requiere que reflexionen y lleven a discusión permanente el impacto de las nuevas tecnologías en la sociedad local y global. Deben planear y promover el uso saludable de las TIC, incluidos los asientos, luz y sonido.

- Cuestiones técnicas: Es un aspecto del tema de aprendizaje permanente a través del cual los profesores deben actualizar sus conocimientos en hardware y software, con base en las nuevas tecnologías que emergen. En concreto, se requiere que los docentes sean capaces de usar y seleccionar, entre una gama de recursos, TIC para mejorar personal y profesionalmente; así como, por propia voluntad, actualizar sus conocimientos a la luz de nuevos desarrollos.

En una investigación que se llevó a cabo en el 2007, que contempló diez centros de educación infantil y primaria con atención a estudiantes con NEE, en Salamanca y Valladolid (España), se pudo constatar, a través de la entrevista a educadores diferenciales, que "las actitudes que manifiestan los profesores sobre el valor de las TIC para el desarrollo curricular en aulas con alumnos de NEE son positivas. Pero esta actitud no va más allá de una mera declaración de intenciones, porque en realidad no están habituados a trabajar con tales materiales y, consecuentemente, carecen de sentido dentro de su práctica profesional habitual (...)" (Ramírez, Domínguez y Clemente, 2007, p. 367). Por otra parte, en lo referente a la categoría "Formación para el uso de las TIC en relación con las NEE", la conclusión es clara: Los docentes la consideran insuficiente, tanto la recibida en su formación inicial como en su desarrollo profesional. 


\subsubsection{Las tecnologías de la información en la Educación Especial}

En este ámbito, sin duda, aún no se está en condiciones de definir cuáles son los aportes de las TIC a las personas con NNE, pues hacen falta más investigaciones y estudios en esta material. No obstante, encuentros académicos como los de Tecnología Educativa y Atención a la Diversidad -Tecnoneet- o en diferentes congresos, como el -TISE- en Chile, confirman que cada vez son más las personas que encuentran en las tecnologías un punto de apoyo para su desarrollo: los alumnos, para compensar discapacidades; y los profesores, para alcanzar su máximo desarrollo profesional y potenciar el desarrollo cognitivo de los alumnos, al mejorar los procesos de adquisición de los objetivos de las distintas disciplinas del currículo escolar (Soto, s. f.).

\section{Metodología}

\subsection{Objetivo general de la investigación}

Analizar la percepción en el uso de recursos tecnológicos dentro de las prácticas pedagógicas, por parte de los profesores de Educación Especial que se desempeñan en grupos diferenciales de la comuna de Chillán, durante el año 2009.

\subsection{Tipo y diseño del estudio}

El tipo de estudio corresponde a un diseño descriptivo. Como plantean Latorre, del Rincón y Arnal (1997), este tipo de diseño pretende describir un fenómeno dado, al analizar su estructura y explorar las asociaciones relativamente estables de las características que lo definen. Para Carmo y Malheiro (2008), este tipo de diseño implica estudiar, comprender y explicar la situación actual del objeto de investigación. Incluye una recolección de datos para probar hipótesis o responder preguntas. Los datos en una investigación descriptiva son normalmente obtenidos mediante la administración de un cuestionario, la realización de entrevistas o a través de la observación de situaciones reales. La información recolectada puede relacionarse con actitudes, opiniones, datos demográficos, condiciones o procedimientos. Dado que para estos estudios son formuladas preguntas que no han sido propuestas con anterioridad o que requieren la recolección de datos que no están disponibles, esta investigación exige frecuentemente la elaboración de instrumentos apropiados para obtener la información necesaria (Carmo y Malheiro, 2008).

\subsection{Definición del grupo participante en la investigación}

\subsubsection{Población y muestra}

Se realizó un estudio preliminar que sirve como base para establecer la población de esta investigación, el cual tuvo por objetivo determinar si el uso del software educativo "Abecedario letrado" (Aburto, 2002), mejoraba la comprensión lectora en estudiantes de 
$3^{\circ}$ y $4^{\circ}$ años en escuelas municipalizadas de la comuna de Chillán, este fue desarrollado entre los años 2007-2008. Para esta primera investigación, las escuelas fueron separadas por nivel socioeconómico, con base en la clasificación del Ministerio de Educación a las escuelas y colegios en el año 2000, para así comparar los resultados obtenidos por estos en el SIMCE (Sistema de Medición de la Calidad de la Educación). Esta categorización “(...) agrupa a los establecimientos educacionales de acuerdo a las características socioeconómicas predominantes de sus alumnos considerando los años promedio de escolaridad del padre y la madre, el ingreso familiar y el índice de vulnerabilidad escolar del establecimiento" (Ministerio de Educación de Chile, Departamento de Estudios y Estadísticas, 2003, p. 4). Se excluyeron del estudio aquellas escuelas municipalizadas que no contaban con Educación Especial. Dado que la población se encontraba dividida en conglomerados naturales (grupo socioeconómico), se realizó una división aleatoria por conglomerado, y se trabajó con toda la población en estudio. Participaron aproximadamente 400 alumnos de ambos sexos y 40 profesores de grupos diferenciales, distribuidos en 29 establecimientos de enseñanza básica municipalizados. Dependiendo de la matrícula, se consideró una división del 50\%, partiéndola en dos grupos donde uno de ellos utilizó el software (experimental) y el otro no (control). Con base en estos antecedentes, la población de estudio de la presente investigación corresponde a los profesores de Educación Especial de la comuna de Chillán, y para esta investigación se ha decidido trabajar con un grupo escogido intencionalmente, que son los profesores de grupos diferenciales que sí utilizaron el software "Abecedario letrado" en el primer estudio, y que, por tanto, tuvieron la capacitación inicial en este. En total participaron 20 de estos profesores.

En otras palabras, el marco referencial de la población de este estudio está dado por los siguientes parámetros:

- Todos los profesores pertenecen a la comuna de Chillán, Chile.

- El número total de profesores de grupos diferenciales de la comuna corresponde a 39, para este estudio se consideraron, intencionalmente, 20 de ellos.

- Para la primera investigación estos 20 profesores fueron capacitados en la utilización del software "Abecedario letrado".

\subsection{Cuestionario}

Para la elaboración del cuestionario aplicado a los profesores de los diferentes grupos diferenciales se tomó como referencia el Instrumento de evaluación del software, propuesto por Aguirre y Martín (1994). Desde el cual se seleccionaron las preguntas relativas a la percepción del uso de tecnología. Quedó finalmente constituido por 2 partes. La primera cuyo objetivo es la obtención de datos relativos a los antecedentes del profesor, para su posterior caracterización, y la segunda, recopilación referida al uso y percepción de recursos tecnológicos en las prácticas pedagógicas de los profesores de grupos diferenciales. 


\subsection{Validez y confiabilidad de los instrumentos}

Una vez definido el cuestionario, se procede a observar los criterios de validez y confiabilidad necesarios a fin de definir si el instrumento mide lo que desea y si puede ser confiable para aplicarse en tiempos y lugares diferentes (Pérez, 2006); por tal razón, el cuestionario fue sometido a una evaluación y análisis por parte de 6 expertos en el área. Como la población de este estudio es reducida $(\mathrm{n}=20)$ se optó por el análisis de expertos, ya que la aplicación de una prueba piloto podría poner en duda la validez de los datos.

Durante el mes de marzo del año 2010, el cuestionario fue aplicado mediante la herramienta 2.0 Google Docs, el cual permite la creación de formularios en línea, enviados mediante correo electrónico y cuyos datos se almacenan en una hoja de cálculo. Los datos fueron exportados al programa SPSS, donde se procedió a realizar el procesamiento de datos con pruebas estadísticas descriptivas, inferenciales y de multivariables.

\section{Resultados y análisis}

\subsection{Datos generales:}

\subsubsection{Promedio de edades profesores de Educación Especial}

La edad promedio de los profesores de Educación Especial de la comuna de Chillán-Chile, es de 51 años, lo que no constituye un dato nuevo, ya que en el año 2008 se realizó un estudio a cargo del Centro de Microdatos (CMD) de la Universidad de Chile, que determinó que el perfil de edad de los profesores es alto. Mientras la edad promedio de los docentes es de 48,2 años, la de los profesionales es de 41,4. Como referencia, el CMD indicó que la edad promedio de la población chilena mayor de 20 años, es de 43. Este fuerte envejecimiento, a juicio de los investigadores, puede estar afectado por la disminución del interés de los jóvenes por estudiar carreras de Pedagogía y por la ley de estatuto docente, que rige los establecimientos municipales y declara la inamovilidad del profesor en el cargo, a menos de que exista un sumario.

Tabla 1

Edades profesores de Educación Especia

\begin{tabular}{|c|c|c|c|c|}
\hline & $\mathrm{N}^{\circ}$ & Mínimo & Máximo & Media \\
\hline Edad & 20 & 34 & 65 & 51 \\
\hline
\end{tabular}

\subsubsection{Promedio en años de servicio de profesores de Educación Especial}

Dentro del grupo de profesores de grupos diferenciales que participó en esta investigación, el rango de años de servicio docente corresponde de 14 a 44 años, con un promedio de 25 años de servicio. Este dato es respaldado por una investigación realizada por la Universidad Católica, encomendada por el Colegio de Profesores A.G. de Chile, en donde se determinó que el profesorado 
estudiado tuvo un promedio de ejercicio laboral docente de 20,5 años, y revela que se trata de una muestra con un grado adecuado de exposición laboral (Colegio de Profesores de Chile, A. G., 2000).

Tabla 2

Años de servicio docente profesores de Educación especial

\begin{tabular}{|l|c|c|c|c|}
\hline & N $^{\circ}$ & Mínimo & Máximo & Media \\
\hline Años de servicio docente & 20 & 14 & 44 & 25 \\
\hline
\end{tabular}

\subsubsection{Cantidad de profesores de Educación Epecial, según sexo}

Del grupo de profesores considerados para esta investigación, el 10\% corresponde a profesionales de sexo masculino y un $90 \%$ al femenino. Esta información se asevera con la obtenida por el estudio del Centro de Microdatos (CMD) de la Universidad de Chile, el cual reveló que la gran mayoría del cuerpo docente del país está compuesto por mujeres, representada por un $73 \%$ del total de docentes.

Tabla 3

Distribución por sexo profesores de Educación Especial

\begin{tabular}{|l|c|c|}
\hline Sexo & Frecuencia & Porcentaje \\
\hline Hombre & 2 & 10 \\
\hline Mujer & 18 & 90 \\
\hline Total & 20 & 100 \\
\hline
\end{tabular}

\subsubsection{Promedio de ordenadores disponibles para el trabajo con estudiantes en Educación Especial}

El 60\% de los grupos diferenciales que participaron de esta investigación tienen un ordenador para trabajar con los estudiantes. Existe un 25\% que dispone de dos ordenadores; un 10\%, de 3 ordenadores y un 5\% que no tiene. Cabe recordar, en esta parte, que en estos grupos se atienden 5 estudiantes como máximo por periodo de clase.

Tabla 4

Distribución de ordenadores disponibles para estudiantes de Educación Especial

\begin{tabular}{|c|c|c|}
\hline Cantidad de ordenadores & Frecuencia & Porcentaje \\
\hline 1 & 12 & 60 \\
\hline 2 & 5 & 25 \\
\hline 3 & 2 & 10 \\
\hline Total & 20 & 100 \\
\hline
\end{tabular}




\subsubsection{Aprendizaje en Informática de los profesores de Educación Especial}

Observamos, a través de los resultados de esta pregunta, que el 60\% de los profesores reconoce que ha sido capacitado a través del proyecto Enlaces, en lo referido al aprendizaje en Informática. Con estos datos se evidencia que el objetivo de este proyecto a nivel ministerial que es apoyar el desarrollo de competencias en tecnologías de la información y la comunicación para la adecuada implementación del currículum, se cumple. Un 35\% reconoce que su aprendizaje ha sido gracias a su esfuerzo personal. Un 5\% considera la opción "otros". Para este caso en particular, el profesor ha aportado la información que él tiene estudios de Informática como analista programador, es decir, no aplicados a Educación, sino como una formación paralela.

Tabla 5

Aprendizaje de Informática de los profesores de Educación Especial

\begin{tabular}{|l|c|c|}
\hline & Frecuencia & Porcentaje \\
\hline Esfuerzo personal & 7 & 35 \\
\hline Cursos ENLACES & 12 & 60 \\
\hline Otros & 1 & 5 \\
\hline Total & 20 & 100 \\
\hline
\end{tabular}

\subsubsection{Motivación por la elección del trabajo en Educación Especial}

Los resultados de esta pregunta nos indican que el 55\% de los profesores de grupos diferenciales, que participaron de esta encuesta, escogieron desarrollar esta profesión docente porque siempre fue de su agrado. El 20\% opina que trabaja en el ámbito de Educación Especial porque corresponde a su especialidad.

El 25\% de los profesores que indicó la opción "otro" aporta que siempre había tenido la intención de trabajar en el área de Educación Especial, pero que como formación inicial tienen otra especialización. Después optaron por un pos título en Educación Especial que los habilitó para trabajar en esta área.

Tabla 6

Elección de la profesión de profesor de Educación Especial

\begin{tabular}{|l|c|c|}
\hline & Frecuencia & Porcentaje \\
\hline Siempre me gustó & 11 & 55 \\
\hline Es mi especialidad & 4 & 20 \\
\hline Otros & 5 & 25 \\
\hline Total & 20 & 100 \\
\hline
\end{tabular}




\subsection{Preguntas cuestionario referidas a la percepción y utilización de recursos tecnológicos dentro de las prácticas pedagógicas}

\subsection{1 ¿Cuál es el nivel de manejo de recursos informático por parte de los profesores de Educación Especial?}

El 65\% del grupo de profesores de grupo diferencial que participaron en este estudio se identifica con un nivel medio en el manejo de recursos informáticos. Un $30 \%$ se sitúa en un nivel alto y un 5\%, en un nivel avanzado. Ningún profesor se identifica con un nivel bajo ni nulo.

\subsection{2 ¿Dentro de sus prácticas pedagógicas considera que la utilización de recursos tecnológicos es importante?}

Un 95\% de los profesores de grupos diferenciales de esta investigación considera que la utilización de recursos tecnológicos dentro de sus prácticas pedagógicas es importante. Un 5\% considera que no lo es.

\subsubsection{Con la incorporación de recursos tecnológicos a la Educación, usted, ¿considera que ha modificado sus prácticas pedagógicas?}

Frente a esta pregunta, un $95 \%$ de los profesores de grupo diferencial dice haber modificado sus prácticas pedagógicas al incluir recursos tecnológicos. Un 5\% dice que no.

\subsection{4 ¿Cuenta con software educativo para trabajar con sus estudiantes?}

El $85 \%$ de los grupos diferenciales cuenta con software educativo para trabajar con los estudiantes que asisten a él. El 15\% dice no contar con ninguno. Los softwares mayormente nombrados por los profesores son:

- Abrapalabra

- Pipo aprende a leer

- Lexia

- Jugando a las Matemáticas

- Gramática

- Primer diccionario interactivo

- Silabario luz

- Conejo lector

- Los dinosaurios

- El árbol mágico del pensamiento

- La feria de los números

- Rompecabezas animados

- Matemágicas

- Matemáticas creativas 


\section{Conclusiones}

La evolución que ha tenido el concepto de necesidad educativa especial ha comprometido a muchas personas que están conscientes y esperanzadas de que se puede lograr la equidad y manejar este tema como algo perteneciente a la sociedad. Cada quien es distinto y en las diferencias está la riqueza de la humanidad.

Se está de acuerdo en que no se puede utilizar la tecnología porque sí, sino que esta debe responder a las necesidades de los estudiantes (estilos de aprendizaje, motivación, madurez, problemas de aprendizaje, entre otros) y del contenido a enseñar. Es clave la planeación de la clase por parte del docente, por ende, es necesaria, por parte de él, una confianza y un conocimiento tecno-pedagógico de recursos tecnológicos. Esto exige una actualización permanente y un cambio radical en la formación inicial del profesorado de educación especial. Ahora si se advierte exclusivamente en la formación tecnológica de este, se puede admitir que no es acorde para nada con las necesidades actuales del entorno educativo, ya que no contempla una incorporación de competencias informáticas en ninguna de las dimensiones establecidas dentro de la formación inicial por el Marco de la Buena Enseñanza (pedagógica, técnica o instrumental, de gestión, social, ética y legal, desarrollo y responsabilidad profesional), ni menos una aplicación de estas al proceso educativo.

Esta investigación ha constatado que los docentes que ejercen en Educación Especial son profesionales en su mayoría mujeres (90\%) y que cuentan con un promedio de edad de 51 años y con un promedio de 25 años de servicio docente, lo que perfila un profesional con bastante experiencia laboral. A pesar de lo que se piensa de los profesionales con muchos años de servicio, todos los profesores participantes en este estudio dicen tener formación en recursos informáticos, lo que posiblemente establece la relación con el alto porcentaje de profesores que considera que la utilización de recursos tecnológicos dentro de sus prácticas pedagógicas es importante (alcanza el 95\%).

Se está consciente de que, a la luz de los resultados de esta investigación, los profesores de Educación Especial reconocen explícitamente que han cambiado sus prácticas docentes al utilizar recursos tecnológicos, ya que estos resultan valiosos e importantes, pero más allá de admitirlo no hay mayores evidencias de su utilización.

Se aprecian, además, los esfuerzos gubernamentales por dotar a los grupos de Educación Especial con recursos tecnológicos, específicamente software educativo, tales como: Abrapalabra (Unlimited, 1998), Pipo aprende a leer (Cibal Multimedia, 1996), Lexia (Stora Sköndalm s. f.), Jugando con las Matemáticas (Royce 2003), Conejo lector (The Learning Company, 1990), El mundo de las mates: La feria de los números (Enterprise Centre, 1996), El árbol mágico del pensamiento (Unlimited, 1995), entre los más destacados y, por formar a los docentes en ejercicio, en la utilización de estos.

Los niños que presentan necesidades educativas especiales son un grupo que con mayor lentitud que el resto ha incorporado el uso de estos recursos dentro de su proceso de enseñanza aprendizaje. Si bien estudios informan que la tecnología es un elemento valioso que aumenta la motivación de estos, existen pocas experiencias en este ámbito. Por tanto, resulta urgente dedicar más esfuerzos y tiempo a ello. 
URL: http://www.una.ac.cr/educare

\section{Referencias bibliográficas}

Aburto, R. (2002). Abecedario Letrado (v.1.0) [CD-ROM]. Chile: Aburto, Raquel.

Aguirre, A. M. y Marín, E. (1994). Indicadores e instrumento de evaluación de la calidad del software educativo. Enseñanza y tecnología, 3, 25-28.

Carmo, H. y Malheiro, M. (2008). Metodologia da investigação. Guia para auto-aprendizagem [Metodología de la Investigación. Guía para el autoaprendizaje]. Lisboa, Portugal: Universidade Aberta.

Chile. Ministerio de Educación, Departamento de Estudios y Estadísticas (2003). Metodología para agrupar establecimientos por nivel socioeconómico. Prueba SIMCE. $4^{\circ}$ Básico 2002. Recuperado de http://www.mineduc.cl/biblio/documento/396_Construccion_de grupos comparables de establecimientos SIMCE 2.pdf

Cibal Multimedia (1996). Pipo Aprende a leer (v.1.0) [CD_ROM]. España: Cibal Multimedia.

Colegio de Profesores de Chile. A. G. (2000). Estudio de la salud laboral de los profesores de Chile. Recuperado de http://www.opech.cl/bibliografico/Doc Docente/Estudio\%20de\%20 Salud\%20Laboral\%20de\%20Profesores\%20en\%20Chile.\%20MINEDUC-PUC.pdf

Costa, F. y Viseu, S. (2008). Formação - Acção - Reflexão: Um modelo de preparação de professores para a integração curricular das TIC [Formación - Acción - Reflexión: Um modelo de preparación para professores para la integración curricular de las TIC]. En F. Costa, H. Peralta y S. Viseu (Eds.). As TIC na Educação em Portugal. Concepções e Práticas [Las TIC en la Educación en Portugal. Concepciones y prácticas] (pp. 238-258). Lisboa: Porto Editora.

Díaz, O. C., Rojas, S. L. y Vasco, E. (2000). La Educación especial en Iberoamérica. (Colección Cuadernos de Educación Comparada, Vol. 2). Madrid: Organización de Estados Iberoamericanos para la Educación, la Ciencia y la Cultura (OEI).

Díaz, V. (2001). Diseño y elaboración de cuestionarios para la investigación comercial. Madrid, España: Esic Editorial.

Enterprise Centre. (1996). El mundo de las mates: La feria de los números (s/v) [CD_ROM]. Irlanda: Enterprise Centre.

Gallego, J. L. y Rodríguez, A. (2007). Tendencias en la formación inicial del profesorado en Educación Especial. Revista electrónica sobre calidad, eficacia y cambio en Educción, 5(3), 102-117. Recuperado de http://dialnet.unirioja.es/servlet/articulo?codigo=2354543\&orden=131 $\underline{719 \& \text { info=link }}$ 
Gento, S. (2003). Educación Especial. Madrid, España: Sanz y Torres.

Gútiez, P. (2003). La evolución histórica de la Educación Especial. En S. Gento (Aut.). Educación Especial (pp. 35-39). Madrid, España: Sanz y Torres.

Latorre, A., Del Rincón, D. y Arnal, J. (1997). Bases metodológicas de la investigación educativa. Barcelona, España: Jordi Hurtado Mompeo.

McClintock, R. (1993). El ordenador como sistema. En R. McClintock, M. J. Streibel y G. Vázquex (aut.). Comunicación tecnología y diseños de instrucción: La construcción del conocimiento escolar y el uso de los ordenadores (pp. 7-32). Madrid: Secretaría general técnica, Centro de Publicaciones.

Ministerio de Educación de Chile [MINEDUC]. (2005). Política Nacional de Educación Especial. Nuestro compromiso con la diversidad ( $2^{\mathrm{a}}$ ed). Santiago, Chile: Autor. Recuperado de http://www.mineduc.cl/usuarios/edu.especial/File/DOCUMENTOS\%20VARIOS\%202008/ POLiTICAEDUCESP.pdf

Ministerio de Educación de Chile [MINEDUC]. (2007). Competencias TIC en la profesión. Recuperado de http://www.enlaces.cl/portales/competenciastic/

Pavone, M. (2009). L'intégration Scolaire des élèves handicapés et la formation des Enseignantes Spécialisés en Italie [La integración escolar de los alumnos discapacitados y la formación de los profesores especialistas en Italia]. Les Sciences de l'éducation, 42(1), 33- 55.

Pérez, L. A. (2006). Marketing social Teoría y Práctica. México: Prentice Hall.

Pérez, L. C. (2003). Discapacidad y exclusión social en la Unión Europea. Tiempo de cambio, herramientas para el cambio (Colección CERMI). España: Comité español de representantes de personas con discapacidad. Recuperado de http://www.infodisclm.com/documentos/ discap exclus social ue.pdf

Prensky, M. (2001). Digital Natives, Digital Immigrants Immigrants [Nativos Digitales, Inmigrantes Digitales]. Recuperado de http://www.marcprensky.com/writing/Prensky - Digital Natives, Digital Immigrants - Part1.pdf

Ramírez, E., Domínguez, A. B. y Clemente, M. (2007, enero-abril). Cómo valoran y usan las tecnologías de la información y la comunicación (TIC) los profesores de alumnos con necesidades educativas especiales (NEE). Revista de educación, 342, 349-372. Recuperado de http://dialnet.unirioja.es/servlet/articulo?codigo=2254203

Royce. (2003). Jugando con las Matemáticas (v1.0) [CD_ROM]. México: Royce. 
Sanches, I. (1995, mayo-junio). Professores de educação especial: Da formação às práticas educativas [Profesores de educación especial: De la formación a las prácticas educativas]. O Professor, $44,44-52$.

Semenov, A. (2006). Las tecnologías de la información y la comunicación en la enseñanza. Manual para docentes o cómo crear nuevos entornos de aprendizaje abierto por medio de las TIC. Organización de las Naciones Unidas, para la Educación, la Ciencia y la Cultura. Montevideo, Uruguay: Ediciones Trilce. Recuperado de unesdoc.unesco.org/ images/0013/001390/139028s.pdf

Soriano, V. (2000). La educación integradora en Europa. Valencia: Artes Gráficas Soler.

Soto, F. (s. f.). Nuevas tecnologías y atención a la diversidad: Oportunidades y retos. Murcia: Dirección general de Enseñanzas Escolares. Servicio de Atención a la Diversidad. Consejería de Educación y Cultura. Recuperado de http://www.niee.ufrgs.br/eventos/CIIEE/2007/pdf/ CE- 143 Espana.pdf

Stora Sköndal. (s. f.). Lexia (v.3.0) [CD_ROM]. Suecia: Stora Sköndal.

The Learning Company (1990). Conejo Lector (v.1.0) [CD_ROM]. Estados Unidos: Nombre del productor.

The Warnock Report (1978). Special educational needs. The History of Education in England [Educación de Necesidades Especiales. La Historia de la Educación en Inglaterra]. Recuperado de http://www.dg.dial.pipex.com/documents/docs3/warnock.shtml

UNESCO. (2002). Information and Communication Technologies in Teacher Education. A Planning Guide [Tecnologías de la información y comunicación en la formación del profesorado]. Recuperado de http://unesdoc.unesco.org/images/0012/001295/129533e.pdf

UNESCO. (2011). A Profile of Inclusive Teachers (Resumen) [El perfil inclusivo de los profesores]. Recuperado de http://www.ibe.unesco.org/fileadmin/user upload/COPs/News documents/2011/1103 Norway/Profile of Inclusive Teachers - summary.pdf

Unlimited. (1995). El árbol mágico del pensamiento (v.1.0) [CD_ROM]. Chile: Unlimited.

Unlimited. (1998). Abrapalabra (v.1.0) [CD_ROM]. Chile: Unlimited.

Vázquez C. M. y Fernández, M. E. (2003). El proyecto de la Agencia Europea sobre la aplicación de las tecnologías de la comunicación e información a la educación del alumnado con necesidades educativas especiales. En F. J. Soto y J. Rodríguez (Coords.). Las nuevas tecnologías en la respuesta educativa a la diversidad (pp. 163-168). España, Murcia: Servicio de ordenación administrativa y publicaciones. Recuperado de http://www.doredin.mec.es/ documentos/018200220032.pdf 